\title{
USEFULNESS OF RANDHAWA AND PUJAHARI SCORING SYSTEM FOR ASSESSMENT OF DIFFICULTY DURING LAPAROSCOPIC CHOLECYSTECTOMY PROCEDURE
}

\author{
Anjani Tripathi ${ }^{1}$, Nisar Ahmad Ansari ${ }^{2}$, Osman Musa $^{3}$, Mamta Dwivedi $^{4}$ \\ ${ }_{1}^{1}$ Resident, Department of General Surgery, Era's Lucknow Medical College, Lucknow, Uttar Pradesh, India. \\ ${ }^{2}$ Associate Professor, Department of General Surgery, Era's Lucknow Medical College, Lucknow, Uttar Pradesh, India. \\ ${ }^{3}$ Professor, Department of General Surgery, Era's Lucknow Medical College, Lucknow, Uttar Pradesh, India. \\ ${ }^{4}$ Senior Resident, Department of General Surgery, KGMU, Lucknow, Uttar Pradesh, India.
}

\section{BACKGROUND}

ABSTRACT

We wanted to evaluate the difficulties encountered during laparoscopic cholecystectomy.

\section{METHODS}

150 adult patients fulfilling the inclusion criteria of study, were enrolled. Using Preoperative predicting score of Randhawa et al. (2009) preoperative score of all the patients was calculated and 99 (66.0\%) patients with pre-operative score of 0-5 were classified as Group I and considered Easy for surgery, rest 51 (34.0\%) patients had pre-operative score 6-10 were classified as Group II and were considered Difficult for surgery. No patients had pre-operative score $>10$ as very difficult for surgery.

\section{RESULTS}

Age of patients ranged between 22-60 years and mean age was $39.62 \pm 11.24$ years and majority of the patients $(60.0 \%)$ were females and were found to be comparable among Easy (Group I) and Difficult (Group II) for surgery. The role of predictive risk factors on the final outcome has been assessed. We found that only previous history of hospitalization, BMI $>27.5 \mathrm{~kg} / \mathrm{m}^{2}$, abdominal scar and pericholecystic collection were significant contributors in prediction of level of ease of surgery. Role of age, male gender, palpability and thickness of GB wall was not found to be significant.

\section{CONCLUSIONS}

This study validated the usefulness of Randhawa and Pujahari scoring system for assessment of difficulty during laparoscopic cholecystectomy and found it comparable to other existing scoring systems. Despite this, a compromised efficacy of different scoring systems to miss difficulty remains a question to be answered. Further studies to identify more variables that could be included to improvise the scoring systems are recommended.

\section{KEY WORDS}

Gallstones, Laparoscopy, Cholecystectomy

HOW TO CITE THIS ARTICLE: Tripathi A, Ansari NA, Musa O, et al. Usefulness of Randhawa and Pujahari scoring system for assessment of difficulty during laparoscopic cholecystectomy procedure. J. Evolution Med. Dent. Sci. 2019;8(27):2166-2170, DOI: $10.14260 /$ jemds/2019/475

\section{BACKGROUND}

Gallstone disease (GSD) is one of the major causes of morbidity and mortality all over the world. Its prevalence in India has been reported 18.8\%.(1) In developed societies too, gallstones constitute a significant health problem, affects $10 \%$ to $15 \%$ of the adult population. (2) Cholecystectomy is done to remove the gallbladder in case of gallstones with acute or chronic cholecystitis and associated complications. first laparoscopic cholecystectomy (LC) was performed in 1985 by Prof. Dr. Erich Mühe of Germany after a time interval of about 100 years when Carl Langenbuch (1846-1901) of Germany performed the first cholecystectomy in 1882.(3) Laparoscopic cholecystectomy has clearly displaced open cholecystectomy in the management of simple biliary lithiasis and is a safe, efficient technique.

'Financial or Other Competing Interest': None.

Submission 15-05-2019, Peer Review 20-06-2019,

Acceptance 26-06-2019, Published 08-07-2019.

Corresponding Author:

Dr. Nisar Ahmad Ansari,

Era's Lucknow Medical College and Hospital,

Sarfarazganj, Lucknow-226003,

Uttar Pradesh, India.

E-mail: nisy1972nisy@yahoo.co.in

DOI: $10.14260 /$ jemds $/ 2019 / 475$

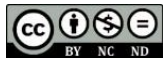

Conversion to open cholecystectomy is occasionally necessary to avoid or repair injury, delineate confusing anatomic relationships, or treat associated conditions. Conversion to open cholecystectomy has been associated with increased overall morbidity, surgical site and pulmonary infections, and longer hospital stays.(4,5) Laparoscopic cholecystectomy is considered to be difficult if adhesions at Calot's triangle are dense, gallbladder is contracted or fibrotic, previous history of upper abdominal surgery, gangrenous gallbladder, acutely inflamed gallbladder, empyema gallbladder including Mirizzi's syndrome, or associated cholecystogastric or cholecystoduodenal fistula.(6) The ability to accurately identify an individual patient's risk for conversion based on preoperative information can result in more meaningful and accurate preoperative counseling, improved operating room scheduling and efficiency, stratification of risks for technical difficulties,(7) and appropriate assignment of resident assistance, may improve patient's safety by minimizing time to conversion, and helps to identify patients in whom a planned open cholecystectomy is indicated. On an average, conversion to open cholecystectomy is required in $2 \%$ to $15 \%$ of patients undergoing laparoscopic cholecystectomy (Alponat et al., 1997; Sanabria et al., 1994).(5,6) It may be helpful to identify difficulties and complications in order to avoid the risk of conversion of a 
laparoscopic cholecystectomy to open cholecystectomy beforehand (Sanabria et al., 1994).(6) Such prediction may allow a surgeon to take extra precautions to reduce intraoperative complications, and to convert from laparoscopic cholecystectomy to open cholecystectomy at an earlier stage.

\section{Aims and Objectives}

The aim of this study is to determine the predictive factors for difficult laparoscopic cholecystectomy and to evaluate the effectiveness of scoring system to predict difficult cholecystectomy.

\section{METHODS}

This prospective study was carried out after clearance from the Institutional Ethical Committee and an informed consent was obtained from all the patients. Sample size was calculated at Department of Social \& Preventive Medicine, Era's Lucknow Medical College \& Hospital on the basis of positive prediction value of scoring method using the formula:

$\mathrm{n}=\mathrm{Z}_{\alpha}^{2} \mathrm{pq} / \mathrm{l}^{2}$

When $\mathrm{p}=75 \%$, proportion of sensitivity.

$\mathrm{q}=100-\mathrm{p}$

Type I error $\mathrm{a}=5 \%$

Allocated even $\mathrm{L}=7.5 \%$ for detecting result with $95 \%$ power of study.

Loss to follow-up $=20 \%$

Then sample size comes out to be $n=150$

150 patients were enrolled for laparoscopic cholecystectomy for cholelithiasis by the following inclusion and exclusion criteria.

\section{Inclusion Criteria}

- $\quad$ Age 18-60 years

- Patients found to have Gall Stone Disease on Abdominal Ultrasonography planned for surgery

\section{Exclusion Criteria}

1. Common bile duct (CBD) calculus.

2. Dilated CBD.

3. Features of obstructive jaundice.

4. Deranged liver function tests.

5. Not willing for laparoscopic cholecystectomy.

6. Contraindication for Laparoscopic Cholecystectomy.

All the patients were evaluated on the basis of Randhawa \& Pujahari, 2009 (8) Scoring system and then predicted the level of difficulty of surgery.

\begin{tabular}{|c|c|c|}
\hline Scoring Factors & Score & Maximum Score \\
\hline \multicolumn{3}{|c|}{ History } \\
\hline $\begin{array}{l}\text { Age (Years) } \\
\quad<50 \\
>50\end{array}$ & $\begin{array}{l}0 \\
1\end{array}$ & 1 \\
\hline $\begin{array}{c}\text { Sex } \\
\text { Female } \\
\text { Male }\end{array}$ & $\begin{array}{l}0 \\
1\end{array}$ & 1 \\
\hline $\begin{array}{c}\text { Previous history of hospitalization } \\
\text { No } \\
\text { Yes }\end{array}$ & $\begin{array}{l}0 \\
4\end{array}$ & 4 \\
\hline \multicolumn{3}{|c|}{$\begin{array}{ll}\text { Clinical } \\
\end{array} \quad$ a } \\
\hline $\begin{array}{c}\text { BMI } \\
<25 \\
25.1-27.5 \\
>27.5\end{array}$ & $\begin{array}{l}0 \\
1 \\
2\end{array}$ & 2 \\
\hline $\begin{array}{c}\text { Abdominal scar } \\
\text { No } \\
\text { Infraumbilical } \\
\text { Supraumbilical }\end{array}$ & $\begin{array}{l}0 \\
1 \\
2\end{array}$ & 2 \\
\hline
\end{tabular}

\begin{tabular}{|c|c|c|}
\hline $\begin{array}{c}\text { Palpable gallbladder } \\
\text { No } \\
\text { Yes }\end{array}$ & $\begin{array}{l}0 \\
1\end{array}$ & 1 \\
\hline \multicolumn{3}{|c|}{ Sonography Findings } \\
\hline $\begin{array}{c}\text { Wall thickness } \\
\text { Thin } \\
\text { Thick }>4 \mathrm{~mm}\end{array}$ & $\begin{array}{l}0 \\
2\end{array}$ & 2 \\
\hline $\begin{array}{c}\text { Pericholecystic collection } \\
\text { No } \\
\text { Yes }\end{array}$ & $\begin{array}{l}0 \\
1\end{array}$ & 1 \\
\hline $\begin{array}{c}\text { Impacted stone } \\
\text { No } \\
\text { Yes }\end{array}$ & $\begin{array}{l}0 \\
1\end{array}$ & 1 \\
\hline Total Score: 15 & & \\
\hline
\end{tabular}

Based upon the above scores for prediction of surgery, patients were divided in three groups: Easy: Score $\leq 5$, Difficult: Score 6-10, Very difficult: Score $>10$. Intra operative findings such as time taken for the surgery, biliary/stone spillage, injury to duct/artery or conversion to open cholecystectomy were noted and assessment of level of ease experienced by the surgeon was evaluated based on following factors (Randhawa and Pujahari) 2009.(8)

\begin{tabular}{|c|c|c|c|}
\hline Factors & Easy & Difficult & Very Difficult \\
\hline Time taken (min) & $<60$ & $60-120$ & $>120$ \\
\hline Bile/stone spillage & - & + & + \\
\hline Injury to duct/artery & - & + duct only & + \\
\hline $\begin{array}{c}\text { Conversion to open } \\
\text { cholecystectomy }\end{array}$ & - & - & + \\
\hline Table 2- Randhawa and Pujahari (2009), Easy and Difficult Scoring \\
Factors.
\end{tabular}

Assessment of level of ease of laparoscopic cholecystectomy procedure was done on the basis of above criteria. Post-operative events such as Drain out, Suture out, biliary leakage, surgical site infection or any other complications, histopathology reports were also recorded. Postoperative cases were followed up for any complications for up to 6 weeks.

\section{Statistical Analysis}

The statistical analysis was done using SPSS (Statistical Package for Social Sciences) Version 15.0 statistical Analysis Software. The values were represented in Number (\%) and Mean \pm SD. The following statistical formulas were used-

1. Chi Square Test: To test relationship between categorical variables.

2. Student ' $t$ ' Test: To test the significance of two means, the student 't' test was used.

3. Level of Significance: "p" is level of significance. $p>0.05$ Not significant. $p<0.05$ Significant.

\section{RESULTS}

A total of 150 patients of gall stone disease fulfilling the inclusion criteria were enrolled in study. Each patient was assessed for risk factors, age, gender, previous history of hospitalization, BMI, presence of supraumbilical/ infraumbilical scar, palpable gall bladder, gall bladder wall thickness, pericholecystic collection and impacted stone and preoperative scoring method to predict the difficulty/ ease of level for performing laparoscopic cholecystectomy. Out of 150 patients, $99(66.0 \%)$ having pre-operative score $0-5$ were classified as Group I defined as Easy laparoscopic cholecystectomy, 51 (34.0\%) patients having score 6-10 were classified as Group II defined as Difficult. None of the patient had pre-operative score $>10$ defined as very difficult. 


\begin{tabular}{|c|c|c|c|c|c|c|}
\hline \multirow{2}{*}{ Age Group } & \multicolumn{2}{|c|}{ Group I (n=99) } & \multicolumn{2}{c|}{ Group II (n=51) } & \multicolumn{2}{c|}{ Total (N=150) } \\
\cline { 2 - 7 } & No. & \% & No. & \% & No. & \% \\
\hline$\leq 30$ yrs. & 29 & 29.29 & 10 & 19.61 & 39 & 26.00 \\
\hline $31-40$ yrs. & 30 & 30.30 & 15 & 29.41 & 45 & 30.00 \\
\hline $41-50$ yrs. & 20 & 20.20 & 20 & 39.22 & 40 & 26.67 \\
\hline $51-60$ yrs. & 20 & 20.10 & 6 & 11.76 & 26 & 17.33 \\
\hline
\end{tabular}

Table 3. Age Distribution of Study Population

Age of patients ranged between 22-60 years in both the groups. Difference in age o patients of above two groups was not found to be statistically significant.

\begin{tabular}{|c|c|c|c|c|c|c|}
\hline \multirow{2}{*}{ Gender } & \multicolumn{3}{|c|}{ Group I (n=99) } & \multicolumn{2}{c|}{ Group II (n=51) } & \multicolumn{2}{c|}{ Total (N=150) } \\
\cline { 2 - 7 } & No. & $\mathbf{\%}$ & No. & $\mathbf{\%}$ & No. & $\mathbf{\%}$ \\
\hline Female & 60 & 60.61 & 30 & 58.82 & 90 & 60.00 \\
\hline Male & 39 & 39.39 & 21 & 41.21 & 60 & 40.00 \\
\hline Male:Female & \multicolumn{3}{|c|}{$1: 0.65$} & \multicolumn{2}{|c|}{$1: 0.70$} & \multicolumn{2}{|c|}{$1: 0.67$} \\
\hline \multicolumn{6}{|c|}{ Table 4. Comparison of Gender } \\
\hline \multicolumn{6}{c}{}
\end{tabular}

Though proportion of males was higher among Group II (41.21\%) as compared to Group I (39.39\%) but this difference was not found to be statistically significant. All the patients enrolled in the study presented with complaints of abdominal pain.

\begin{tabular}{|c|c|c|c|c|c|c|c|c|}
\hline & \multicolumn{2}{|c|}{$\begin{array}{l}\text { Group I } \\
(n=99)\end{array}$} & \multicolumn{2}{|c|}{$\begin{array}{c}\text { Group II } \\
(n=51)\end{array}$} & \multicolumn{2}{|c|}{$\begin{array}{c}\text { Total } \\
(\mathrm{N}=150)\end{array}$} & \multicolumn{2}{|c|}{$\begin{array}{c}\text { Statistical } \\
\text { Significance }\end{array}$} \\
\hline & No. & $\%$ & No. & $\%$ & No. & $\%$ & $x^{2}$ & $\mathbf{p}$ \\
\hline Pallor & 2 & 2.02 & 1 & 1.96 & 3 & 2.00 & 0.001 & 0.980 \\
\hline $\begin{array}{l}\text { Abdominal } \\
\text { scar }\end{array}$ & 2 & 2.02 & 34 & 66.67 & 36 & 24.00 & 77.122 & $<0.001$ \\
\hline Tenderness & 5 & 5.05 & 5 & 9.80 & 10 & 6.67 & 1.222 & 0.269 \\
\hline Palpable GB & 10 & 10.10 & 7 & 13.73 & 17 & 11.33 & 0.440 & 0.507 \\
\hline
\end{tabular}

of these presenting symptoms, difference was found to be statistically significan between the above two groups for incidence of abdominal scar $(\mathrm{p}<0.001)$.

\begin{tabular}{|c|c|c|c|c|c|c|c|c|}
\hline & \multicolumn{2}{|c|}{$\begin{array}{l}\text { Group I } \\
(n=99)\end{array}$} & \multicolumn{2}{|c|}{$\begin{array}{l}\text { Group II } \\
(n=51)\end{array}$} & \multicolumn{2}{|c|}{$\begin{array}{c}\text { Total } \\
(\mathrm{N}=150)\end{array}$} & \multicolumn{2}{|c|}{\begin{tabular}{|c|} 
Statistical \\
Significance
\end{tabular}} \\
\hline & No. & $\%$ & No. & $\%$ & No. & $\%$ & $\chi^{2}$ & $\mathbf{p}$ \\
\hline \multicolumn{9}{|c|}{ Calculi } \\
\hline Single, large & 29 & 29.29 & 20 & 39.22 & 49 & 32.67 & \multirow[b]{2}{*}{1.507} & \multirow[b]{2}{*}{0.220} \\
\hline $\begin{array}{c}\text { Small, } \\
\text { multiple }\end{array}$ & 70 & 70.71 & 31 & 60.78 & 101 & 67.33 & & \\
\hline \multicolumn{9}{|c|}{ GB Wall } \\
\hline Normal & 85 & 85.86 & 34 & 66.67 & 119 & 79.33 & \multirow{2}{*}{7.562} & \multirow{2}{*}{0.006} \\
\hline Thickened & 14 & 14.14 & 17 & 33.33 & 31 & 20.67 & & \\
\hline \multicolumn{9}{|c|}{ CBD } \\
\hline Dilated & 3 & 3.03 & 0 & 0.00 & 3 & 2.00 & \multirow{3}{*}{1.672} & \multirow{3}{*}{0.433} \\
\hline Mild dilated & 11 & 11.11 & 5 & 9.80 & 16 & 10.67 & & \\
\hline Normal & 85 & 85.86 & 46 & 90.20 & 131 & 87.33 & & \\
\hline \multicolumn{9}{|c|}{ Pericholecystic collection } \\
\hline Present & 7 & 7.07 & 9 & 17.65 & 16 & 10.67 & 3.951 & 0.047 \\
\hline \multicolumn{9}{|c|}{ IHBRD } \\
\hline Absent & 93 & 93.94 & 48 & 94.11 & 141 & 94.00 & \multirow{2}{*}{0.570} & \multirow{2}{*}{0.903} \\
\hline Present & 6 & 6.06 & 3 & 5.88 & 9 & 0.60 & & \\
\hline
\end{tabular}

There was no statistical difference between two groups on ultrasonographic finding number of calculi, GB wall thickness, CBD dilatation, IHBRD and pericholecystic fluid collection.

\begin{tabular}{|c|c|c|c|c|c|c|}
\hline \multirow{2}{*}{ Variable } & \multicolumn{2}{|c|}{ Group I (n=99) } & \multicolumn{2}{c|}{ Group II (n=51) } & \multicolumn{2}{c|}{ Student 't' test } \\
\cline { 2 - 8 } & Mean & SD & Mean & SD & 't' & 'p' \\
\hline Hb & 10.75 & 2.05 & 10.78 & 1.98 & -0.068 & 0.946 \\
\hline S. Bilirubin & 0.59 & 0.32 & 0.66 & 0.34 & -1.194 & 0.234 \\
\hline SGPT & 49.15 & 5.08 & 50.08 & 3.92 & -1.139 & 0.256 \\
\hline SGOT & 34.76 & 4.11 & 34.04 & 3.85 & 1.036 & 0.302 \\
\hline PT & 12.61 & 1.48 & 12.25 & 1.48 & 1.374 & 0.171 \\
\hline
\end{tabular}

Table 7. Comparison of Biochemical/Haematological Variables among Study Population

Above biochemical/haematological variables of Group I and Group II were found to be comparable.

\begin{tabular}{|c|c|c|c|c|c|c|c|c|}
\hline & \multicolumn{2}{|c|}{$\begin{array}{l}\text { Group I } \\
(n=99)\end{array}$} & \multicolumn{2}{|c|}{$\begin{array}{c}\text { Group II } \\
(n=51)\end{array}$} & \multicolumn{2}{|c|}{$\begin{array}{c}\text { Total } \\
(\mathrm{N}=150)\end{array}$} & \multirow[t]{2}{*}{$\chi^{2}$} & \multirow[t]{2}{*}{$\mathbf{p}$} \\
\hline & No. & $\%$ & No. & $\%$ & No. & $\%$ & & \\
\hline \multicolumn{7}{|c|}{ BMI } & & \\
\hline$\leq 25$ & 14 & 14.14 & 2 & 3.92 & 16 & 16.67 & \multirow{3}{*}{7.095} & \multirow{3}{*}{0.029} \\
\hline $25.1-27.5$ & 32 & 32.32 & 11 & 21.57 & 43 & 28.67 & & \\
\hline$>27.5$ & 53 & 53.53 & 38 & 74.51 & 91 & 60.67 & & \\
\hline \multicolumn{9}{|c|}{ Previous Hospitalization } \\
\hline $\begin{array}{c}\text { No h/o } \\
\text { hospitalization }\end{array}$ & 95 & 95.95 & 2 & 3.92 & 97 & 64.67 & \multirow{2}{*}{124.791} & \multirow{2}{*}{$<0.001$} \\
\hline $\begin{array}{c}\mathrm{H} / \mathrm{o} \\
\text { hospitalization }\end{array}$ & 4 & 4.04 & 49 & 96.08 & 53 & 35.33 & & \\
\hline \multicolumn{9}{|c|}{ Table 8. Comparison of BMI and History of Previous Hospitalization } \\
\hline $\begin{array}{l}\text { ortion of } \\
51 \% \text { ) as co }\end{array}$ & t & $\mathrm{h} \mathrm{Bl}$ & & $\mathrm{g} / \mathrm{m}^{2}$ & & & & \\
\hline
\end{tabular}

\begin{tabular}{|c|c|c|c|c|c|c|c|c|}
\hline & \multicolumn{2}{|c|}{$\begin{array}{l}\text { Group I } \\
(n=99)\end{array}$} & \multicolumn{2}{|c|}{$\begin{array}{c}\text { Group II } \\
(n=51)\end{array}$} & \multicolumn{2}{|c|}{$\begin{array}{c}\text { Total } \\
(\mathrm{N}=150)\end{array}$} & \multirow[t]{2}{*}{$\chi^{2}$} & \multirow[t]{2}{*}{$\mathbf{p}$} \\
\hline & No. & $\%$ & No. & $\%$ & No. & $\%$ & & \\
\hline \multicolumn{7}{|c|}{ Duration of surgery } & \multirow{4}{*}{76.972} & \\
\hline$<60 \min$ & 87 & 87.87 & 8 & 15.69 & 95 & 63.33 & & \multirow{3}{*}{$<0.001$} \\
\hline $60-120 \mathrm{~min}$ & 12 & 12.12 & 37 & 72.55 & 49 & 32.67 & & \\
\hline$>120 \mathrm{~min}$ & 0 & 0.00 & 6 & 11.76 & 6 & 4.00 & & \\
\hline \multicolumn{7}{|c|}{ Adhesions } & \multirow{4}{*}{53.712} & \\
\hline No adhesions & 82 & 82.83 & 11 & 21.57 & 93 & 62.00 & & \multirow{3}{*}{$<0.001$} \\
\hline Flimsy adhesion & 15 & 15.15 & 34 & 66.67 & 49 & 32.67 & & \\
\hline Dense adhesions & 2 & 2.02 & 6 & 11.76 & 8 & 5.33 & & \\
\hline $\begin{array}{l}\text { Difficult Calot's } \\
\text { dissection }\end{array}$ & 9 & 9.09 & 8 & 15.69 & 17 & 11.33 & 1.457 & 0.227 \\
\hline Bile spillage & 7 & 7.07 & 12 & 23.53 & 19 & 12.67 & 8.243 & 0.004 \\
\hline $\begin{array}{l}\text { Cystic artery } \\
\text { injury }\end{array}$ & 0 & 0.00 & 0 & 0.00 & 0 & 0.00 & - & 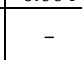 \\
\hline CBD Injury & 0 & 0.00 & 0 & 0.00 & 0 & 0.00 & - & - \\
\hline \multicolumn{9}{|c|}{ Table 9. Comparison of Intraoperative Events } \\
\hline
\end{tabular}

In terms of difference in duration of surgery, adhesions, bile spillage among two groups were found to be highly significant statistically. Out of 150 cases enrolled in the study only $6(4.00 \%)$ of cases converted into open surgery. Proportion of conversion to open surgery was higher among Group II $(11.76 \%)$ as compared to Group I $(0.00 \%)$ Difference in conversion rate to open surgery among patients of above two groups was found lto be statistically significant.

\begin{tabular}{|c|c|c|c|c|c|c|c|c|}
\hline & \multicolumn{2}{|c|}{$\begin{array}{l}\text { Group I } \\
(n=99)\end{array}$} & \multicolumn{2}{|c|}{$\begin{array}{c}\text { Group II } \\
(n=51)\end{array}$} & \multicolumn{2}{|c|}{$\begin{array}{c}\text { Total } \\
(\mathrm{N}=150)\end{array}$} & \multicolumn{2}{|c|}{$\begin{array}{c}\text { Statistical } \\
\text { Significance }\end{array}$} \\
\hline & No. & $\%$ & No. & $\%$ & No. & $\%$ & $\chi^{2}$ & 'p' \\
\hline \multicolumn{9}{|c|}{ Drain Out } \\
\hline POD1 & 52 & 52.53 & 31 & 60.78 & 83 & 55.33 & \multirow{4}{*}{3.443} & \multirow{4}{*}{0.328} \\
\hline POD2 & 36 & 36.36 & 16 & 31.37 & 52 & 34.67 & & \\
\hline POD3 & 3 & 3.03 & 3 & 5.88 & 6 & 4.00 & & \\
\hline POD4 & 8 & 8.08 & 1 & 1.96 & 9 & 6.00 & & \\
\hline \multicolumn{9}{|c|}{ Suture Out } \\
\hline POD7 & 68 & 68.69 & 36 & 70.59 & 104 & 69.33 & \multirow{4}{*}{2.770} & \multirow{4}{*}{0.428} \\
\hline POD8 & 20 & 20.20 & 12 & 23.53 & 32 & 21.33 & & \\
\hline POD9 & 6 & 6.06 & 3 & 5.88 & 9 & 6.00 & & \\
\hline P0D12 & 5 & 5.05 & 0 & 0.00 & 5 & 3.33 & & \\
\hline \multicolumn{9}{|c|}{ SSI } \\
\hline Absent & 94 & 94.95 & 51 & 100.00 & 145 & 96.67 & \multirow{2}{*}{2.665} & \multirow{2}{*}{0.103} \\
\hline Present & 5 & 5.05 & 0 & 0.00 & 5 & 3.33 & & \\
\hline \multicolumn{9}{|c|}{ Table 10. Comparison of Post-operative Events } \\
\hline
\end{tabular}

Difference in post-operative events i.e. Drain out, suture out among cases of Group and Group II was not found to be statistically significant. Incidence of SSI was $3.33 \%$ $(n=5)$ among overall study population. SSI was observed in none of the cases of Group II.

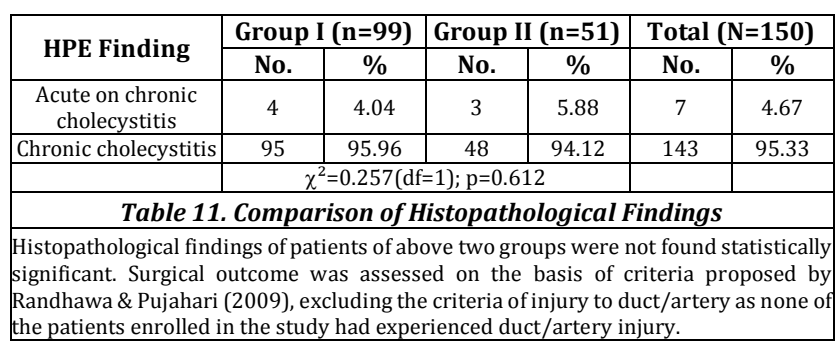

\begin{tabular}{|c|c|c|c|c|c|c|}
\hline \multirow{2}{*}{ Surgical Outcome } & \multicolumn{2}{|c|}{ Group I (n=99) } & \multicolumn{2}{|c|}{ Group II $(n=51)$} & \multicolumn{2}{|c|}{ Total $(\mathrm{N}=150)$} \\
\hline & No. & $\%$ & No. & $\%$ & No. & $\%$ \\
\hline Easy & 87 & 87.87 & 10 & 19.61 & 97 & 64.67 \\
\hline Difficult & 12 & 12.12 & 35 & 68.63 & 47 & 31.33 \\
\hline Very Difficult & 0 & 0.00 & 6 & 11.76 & 6 & 4.00 \\
\hline & \multicolumn{4}{|c|}{$\chi^{2}=70.208(\mathrm{df}=2) ; \mathrm{p}<0.001$} & & \\
\hline
\end{tabular}

Despite low preoperative score of 0-5 (Group I) among 99 patients, the operatin surgeon experienced difficulties that were graded as difficult in $12(12.12 \%)$ patients Similarly, for predictive score of 6-10 (Group II), $6(11.76 \%)$ patients were graded a very difficult to operate, $10(19.61 \%)$ were Easy to operate and rest $35(68.63 \%)$ patients were difficult to operate.

\begin{tabular}{|c|c|c|c|c|c|c|c|}
\hline \multirow{2}{*}{$\begin{array}{l}\text { Predictive Risk } \\
\text { Factors }\end{array}$} & \multirow{2}{*}{$\begin{array}{c}\text { Total } \\
(n=144)\end{array}$} & \multicolumn{2}{|c|}{$\begin{array}{c}\text { Easy } \\
(n=97)\end{array}$} & \multicolumn{2}{|c|}{$\begin{array}{l}\text { Difficult } \\
(n=47)\end{array}$} & \multicolumn{2}{|c|}{$\begin{array}{c}\text { statistical } \\
\text { significance }\end{array}$} \\
\hline & & No. & $\%$ & No. & $\%$ & $\chi^{2}$ & p \\
\hline Age $>50$ years & 25 & 19 & 19.59 & 6 & 12.77 & 1.027 & 0.311 \\
\hline Male gender & 58 & 34 & 35.05 & 24 & 51.06 & 3.375 & 0.066 \\
\hline $\begin{array}{l}\text { Prev. history of } \\
\text { Hospitalization }\end{array}$ & 47 & 14 & 14.43 & 33 & 70.21 & 44.804 & $<0.001$ \\
\hline \multicolumn{8}{|c|}{ BMI $\left(\mathrm{kg} / \mathrm{m}^{2}\right)$} \\
\hline$<25$ & 16 & 14 & 14.43 & 2 & 4.26 & \multirow{3}{*}{6.501} & \multirow{3}{*}{0.039} \\
\hline $25.1-27.5$ & 41 & 31 & 31.96 & 10 & 21.28 & & \\
\hline$>27.5$ & 87 & 52 & 53.61 & 35 & 74.47 & & \\
\hline Abdominal scar & 30 & 9 & 9.28 & 21 & 44.68 & 24.059 & $<0.001$ \\
\hline
\end{tabular}




\begin{tabular}{|c|c|c|c|c|c|c|c|}
\hline Palpable GB & 17 & 8 & 8.25 & 9 & 19.15 & 3.614 & 0.057 \\
\hline Thick GB Wall & 30 & 17 & 17.53 & 13 & 27.66 & 1.971 & 0.160 \\
\hline $\begin{array}{c}\text { Pericholecystic } \\
\text { collection }\end{array}$ & 16 & 7 & 7.22 & 9 & 19.15 & 4.564 & 0.033 \\
\hline
\end{tabular}

Table 13. Association of Easy and Difficult Outcome with Risk Factors

The table above shows the comparison between predictive risk factors with the fina outcome. Surgical outcome was Easy in 97 cases and difficult in 47 cases. Very difficult 6 cases were excluded.

\begin{tabular}{|c|c|c|c|c|c|c|c|}
\hline \multirow{2}{*}{$\begin{array}{l}\text { Predictive Risk } \\
\text { Factors }\end{array}$} & \multirow{2}{*}{$\begin{array}{c}\text { Total } \\
(n=150)\end{array}$} & \multicolumn{2}{|c|}{$\begin{array}{c}\text { Easy/Difficult } \\
(\mathrm{n}=144)\end{array}$} & \multicolumn{2}{|c|}{$\begin{array}{c}\text { Very Difficult } \\
(n=6)\end{array}$} & \multicolumn{2}{|c|}{$\begin{array}{c}\text { Statistical } \\
\text { Significance }\end{array}$} \\
\hline & & No. & $\%$ & No. & $\%$ & $\chi^{2}$ & p \\
\hline Age $>50$ years & 26 & 25 & 17.36 & 1 & 16.67 & 0.002 & 0.965 \\
\hline Male gender & 60 & 58 & 40.28 & 2 & 33.33 & 0.116 & 0.734 \\
\hline H/o Hospitalization & 53 & 47 & 32.64 & 6 & 100.00 & 11.439 & 0.001 \\
\hline \multicolumn{8}{|c|}{ BMI $\left(\mathrm{kg} / \mathrm{m}^{2}\right)$} \\
\hline$<25$ & 16 & 16 & 11.11 & 0 & 0.00 & \multirow{3}{*}{0.751} & \multirow{3}{*}{0.687} \\
\hline $25.1-27.5$ & 43 & 41 & 28.47 & 2 & 33.33 & & \\
\hline$>27.5$ & 91 & 87 & 60.42 & 4 & 66.67 & & \\
\hline Abdominal scar & 36 & 30 & 20.83 & 6 & 100.00 & 15.689 & $<0.001$ \\
\hline Palpable GB & 17 & 17 & 11.81 & 0 & 0.00 & 0.056 & 0.813 \\
\hline Thick GB Wall & 31 & 30 & 20.83 & 1 & 16.67 & 0.000 & 1.000 \\
\hline $\begin{array}{l}\text { Pericholecystic } \\
\text { collection }\end{array}$ & 16 & 16 & 11.11 & 0 & 0.00 & 0.036 & 0.850 \\
\hline
\end{tabular}

Table 14. Role of Risk Factors in Prediction of Very Difficult Outcome

Out of 8 factors for prediction of Very difficult surgery, only 2 i.e. hospital stay and Abdominal scar were found significant contributing factors in predicting the outcome of surgery.

\section{DISCUSSION}

Cholecystectomy can be performed either through open procedure or through laparoscopic procedure. However, laparoscopic cholecystectomy is often criticized as in few patients- conversion to open cholecystectomy, increased risk of bile duct injury or injury to the adjoining viscera and its high cost. Radhawa and Pujahari(8) came up with a systematic scoring system to predict the level of difficulty in laparoscopic cholecystectomy. This scoring system takes into account the demographic factors like age, gender, hospitalization history, clinical factors like BMI, presence of abdominal scar, palpability of gall bladder and sonographic features like wall thickness, pericholecystic collection and impacted stone as the predictors of difficulty. In present study, we also made an attempt to predict the difficulty level in laparoscopic cholecystectomy procedures included in our study and also studied the effectiveness of the scoring system proposed by Randhawa and Pujahari.(8) For this purpose, a total of 150 patients scheduled to undergo laparoscopic cholecystectomy procedure were enrolled in the study. Age of patients ranged from 22 to 60 years with mean age of $39.62 \pm 11.24$ years. The sampled population was dominated by females $(60 \%)$. Yolet al.(9) showed proportion of females to be $50.5 \%$ and mean age as $39.2 \%$. Randhawa et al.(8) in their study had $64.9 \%$ females and reported the mean age of patients as 44.37 years. The findings in general suggest a predominance of females and middle age group as the representative age domain. In present study, a total of $36(24 \%)$ patients had abdominal scar and except for 16 (12.67\%) patients, all the patients had BMI>25 $\mathrm{kg} / \mathrm{m}^{2}$. History of previous hospitalization was positive in $35.33 \%$ patients, a total of $11.33 \%$ patients had palpable gall bladder. Thickened wall was seen in $31(20.67 \%)$ patients and pericholecystic collection in $16(10.67 \%)$ cases. None of the cases had impacted stone. Correspondingly, the difficulty level was assessed as easy in $99(66 \%)$ cases and difficult in 51 (33\%) cases. None of the cases were defined as very difficult. Compared to present study, Randhawa and Pujhari (8) in their study found $78.1 \%$ of their cases as easy and only $21.2 \%$ as difficult. Using a different scoring system based on ultrasonographic features only Lal et al.(10) found $71.23 \%$ of their cases as easy and remaining $28.77 \%$ as difficult. Chand et al.(11) There was a significant association between predicted difficulty and duration of surgery, presence of adhesions during surgery, bile spillage and conversion to surgery. Thus, validating the usefulness of scoring system for these outcomes. The scoring system did not show any association with postoperative complications and outcomes. Thus, implying that the scoring system was meant exclusively for prediction of intraoperative events and difficulties only. Neither Randhawa and Pujahri(8) nor Gupta et al.(12) who developed and validated the scoring system used in present study reported the usefulness of post-operative outcomes and events as the outcomes to be predicted by the scoring system. In present study, no significant association between histopathological findings and predicted difficulty was observed. No such association has been reported in previous studies too. In this study, during surgery, a total of 97 (64.67\%) procedures were found easy, 47 (31.33\%) were difficult and 6 (4\%) were found very difficult. On validating the observed difficulty, we found that out of 97 cases found easy - a total of 87 (89.7\%) were predicted as easy while $10(10.3 \%)$ were predicted as difficult. Out of 47 procedures found difficult, 35 (74.5\%) were predicted to be difficult while $12(25.5 \%)$ were predicted to be easy. All the cases found very difficult were predicted as difficult during prediction. Similar to present study, Gupta et al.(12) reported that out of 141 cases found to be easy, a total of $135(95.7 \%)$ were predicted as easy, out of 57 cases found to be difficult, a total of $42(73.7 \%)$ and all the 12 cases found to be very difficult were predicted to be difficult. An evaluation of all these scoring systems showed that very difficult cases are often missed by these scoring systems. The proportion of missed cases (very difficult) was $4.5 \%$ in present study as compared to $4.4 \%$ in the study of Randhawa and Pujahari(8) and $5.7 \%$ in the study of Gupta et al.(12) Incidentally, in all the series none of the cases were predicted to be very difficult, thus raising the question mark over the criteria used for differentiation. In the present study association of presence of abdominal scar had been found to be a significant factor in predicting difficult outcome of surgery and was similar to findings of Agarwal et al (2015) and contrary to that of study by Acharya and Adhikari, 2012(13) who did not find any association of difficulty level with presence of abdominal scar. In present study, we made an attempt to determine the performance of the scoring system with difficulty (merging difficult and very difficult) as the outcome it was found $77.8 \%$ sensitive and $87.9 \%$ specific. An overview of predictive efficacy of the scoring system used in present study and previous studies is as follows:

\begin{tabular}{|c|c|c|c|c|c|c|c|}
\hline $\begin{array}{l}\dot{z} \\
\dot{m}\end{array}$ & 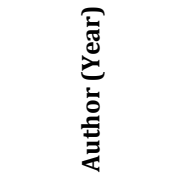 & $\begin{array}{l}\text { जू } \\
\text { के } \\
\frac{0}{2} \\
\text { हूँ }\end{array}$ & 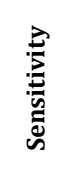 & 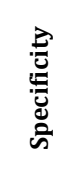 & a & $\frac{\vec{a}}{\mathbf{z}}$ & 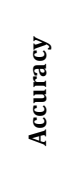 \\
\hline 1. & $\begin{array}{c}\text { Randhawa and } \\
\text { Pujahari (2009)(8) }\end{array}$ & 228 & $68.8 \%$ & $96.3 \%$ & $88 \%$ & $88.8 \%$ & $88.6 \%$ \\
\hline 2. & $\begin{array}{c}\text { Gupta et al. } \\
(2013)^{(12)}\end{array}$ & 210 & $78.3 \%$ & $95.7 \%$ & $90 \%$ & $90 \%$ & $90.0 \%$ \\
\hline
\end{tabular}




\begin{tabular}{|l|c|c|c|c|c|c|c|}
\hline 3. & $\begin{array}{c}\text { Acharya and Adhikari } \\
(2012)^{(13) \#}\end{array}$ & 114 & $71.4 \%$ & $97.2 \%$ & $93.8 \%$ & $85.4 \%$ & $87.7 \%$ \\
\hline 4. & Present study (2018) & 150 & $77.8 \%$ & $87.9 \%$ & $74.5 \%$ & $89.7 \%$ & $84.7 \%$ \\
\hline An Overview of Predictive Efficacy of Randhawa and Pujahari(8) \\
Scoring System for Prediction of Difficulty*
\end{tabular}

This shows that the scoring system lacked equivalent sensitivity and specificity. The role of predictive risk factors on final outcome had been assessed in the present study. We found that only previous history of hospitalization, BMI $>27.5$ $\mathrm{kg} / \mathrm{m}^{2}$, abdominal scar and pericholecystic collection were significant contributors in prediction of level of ease of surgery. Role of age, male gender, palpability and thickness GB wall was not found to be significant. BMI $>27.5 \mathrm{~kg} / \mathrm{m}^{2}$ had been found to be associated with difficulty in surgery which had been supported by Hutchinson et al. (1994),(14) Nachnani \& Supe (2005)(15) and Bouarfa et al. (2011).(16) Association of pericholecystic collection in prediction of difficulty in laparoscopic cholecystectomy has been found in the present study. Study done by Nidoniet al. 2015(17) also supported this finding. The question now arises as to why do these predictive scoring systems fail? One of the obvious reasons is that difficulty during a surgical procedure is a multifactorial issue and to identify and assign integer values to different factors is quite difficult. Moreover, most of the times the difficulty is assessed in terms of patient characteristics, however, one must understand that the difficulty during the operative procedure is an interaction of patient, surgeon and the facilities available.

\section{CONCLUSIONS}

The overall diagnostic accuracy of preoperative predicting score was found to be $84.7 \%$. In our set up previous history of hospitalization, BMI $>27.5 \mathrm{~kg} / \mathrm{m}^{2}$, abdominal scar and pericholecystic collection were found to be significantly contributing in predicting the outcome of surgery. In prediction of very difficult cases, only hospital stay and abdominal scar were found to be significantly contributing in predicting the outcome of surgery. The present study validated the usefulness of Randhawa and Pujahari scoring system for assessment of difficulty during laparoscopic cholecystectomy procedure and found it to be comparable to other existing scoring systems. Despite this, a compromised efficacy of different scoring systems to miss difficulty remains a question to be answered. Further studies to identify more variables that could be included to improvise the scoring systems are recommended. Moreover, one must also understand that skill acquisition to tackle the difficulties is one of the most important parts of learning in medical field. The difficulties should be identified, solved and the technique should be improvised through new techniques, new instrumentations and acquisition of newer skills. It seems that different scoring systems tend to use relaxed criteria for assessment of difficulty that needs to be modified. Further studies for evolution of newer scoring systems are recommended is future.

\section{REFERENCES}

[1] Singh, Trikha B, Nain C, et al. Epidemiology of gallstone disease in Chandigarh: a community-based study. J Gastroenterol Hepatol 2001;16(5):560-3.
[2] Everhart JE, Khare M, Hill M, et al. Prevalence and ethnic differences in gallbladder disease in the United States. Gastroenterology 1999;117(3):632-9.

[3] Litynski GS. Highilghts in the history of laparoscopy. Frankfurt, Germany: Barbara Bernert Verlag 1996: p. 165-8.

[4] Livingston EH, Rege RV. A nationwide study of conversion from laparoscopic to open cholecystectomy. Am J Surg 2004;188(3):205-11.

[5] Alponat A, Kum CK, Koh B, et al. Predictive factors for conversion of laparoscopic cholecystectmy. World J Surg 1997;21(6):629-33.

[6] Sanbria JR, Gallinger S, Croxford R, et al. Risk factors in elective laparoscopic cholecystectomy for conversion to open cholecystectomy. J Am Coll Surg 1994;179(6):696-704.

[7] Santambrogio R, Montorsi M, Bianchi P, et al. Technical difficulties and complications during laparoscopic cholecystectomy: predictive use of preoperative ultrasonography. World J Surg 1996;20(8):197-82.

[8] Randhawa JS, Pujahari AK. Preoperative prediction of difficult lap chole: a scoring method. Indian J Surg 2009;71(4):198-201.

[9] Yol S, Kartal A, Vatansev C, et al. Sex as a factor in conversion from laparoscopic cholecystectomy to open surgery. J SLS 2006;10(3):359-63.

[10] Lal P, Agarwal PN, Malik VK, et al. A difficult laparoscopic cholecystectomy that requires conversion to open procedure can be predicted by preoperative ultrasonography. JSLS 2002;6(1):59-63.

[11] Chand P, Singh R, Singh B, et al. Preoperative ultrasonography as a predictor of difficult laparoscopic cholecystectomy that requires conversion to open procedure. Nigerian Journal of Surgery: Official Publication of the Nigerian Surgical Research Society 2015;21(2):102-5.

[12] Gupta N, Ranjan G, Arora MP, et al. Validation of a scoring system to predict difficult laparoscopic cholecystectomy. International Journal of Surgery 2013;11(9):1002-6.

[13] Acharya A, Adhikari SK. Preoperative scoring system to predict difficulty laparoscopic cholecystectomy. Postgraduate Journal of NAMS 2012;12(1):45-50.

[14] Hutchinson CH, Traverso LW, Lee FT. Laparoscopic cholecystectomy. Do preoperative factors predict the need to convert to open? Surg Endosc 1994;8(8):87580.

[15] Nachnani J, Supe A. Pre-operative prediction of difficult laparoscopic cholecystectomy using clinical and ultrasonography parameters. Indian J Gastroenterol 2005;24(1):16-8.

[16] Bouarfa L, Schneider A, Feussner H, et al. Prediction of intraoperative complexity from preoperative patient data for laparoscopic cholecystectomy. Artif Intell Med 2011;52(3):169-76.

[17] Nidoni R, Udachan TV, Sasnur P, et al. Prediction difficulty laparoscopic cholecystectomy based on clinicoradiological assessment. Journal of Clinical and Diagnostic Research 2015;9(12):PC09-PC12. 